\title{
Determine Joint Factors that Affect Maternal Weight and Body Mass Index Among Pregnant Women in Ethiopia: A Bi-variate Analysis
}

\author{
Melkamu Ayana Zeru*, Kindu Kebede Gebre \\ Department of Statistics, College of Computing and Informatics, Haramaya University, Harar, Ethiopia \\ Email address: \\ melkamu.ayana@gmail.com (M. A. Zeru),m7.kebede@gmail.com (K. K. Gebre) \\ ${ }^{*}$ Corresponding author \\ To cite this article: \\ Melkamu Ayana Zeru, Kindu Kebede Gebre. Determine Joint Factors that Affect Maternal Weight and Body Mass Index Among Pregnant \\ Women in Ethiopia: A Bi-variate Analysis. American Journal of Theoretical and Applied Statistics. Vol. 8, No. 6, 2019 , pp. $214-220$. \\ doi: 10.11648/j.ajtas.20190806.13
}

Received: August 5, 2019; Accepted: October 15, 2019; Published: November 8, 2019

\begin{abstract}
Introduction: A low maternal body mass index and sub-optimal weight gain during pregnancy are long recognized risk factors for delivery of infants too small for gestational age, low birth weight as well as to increase the risk of subsequent obesity and hypertension in the off- spring. Maternal body mass index and maternal weight is positively associated with infant obesity risk. The main objective of this research was to determine the determinants of maternal body mass index and maternal weight simultaneously based on Ethiopia demographic health survey 2016 which was implemented in statistical package R. Methodology: Cross sectional study design was used from Ethiopia demographic health survey 2016. Bi-variate linear regression model was used to determine the factors that affect maternal body mass index and maternal weight simultaneously. Result: The bi-variate analysis of maternal pregnancy weight and body mass index identified that the co-variate husband educational level, preferred waiting time for birth, region, family size, frequency of watching television, maternal height, desire for more children and number of tetanus injections before pregnancy were statistically associated with maternal pregnancy weight. Moreover, educational level of husband, preferred waiting time for birth, region, family size, desire for more children, frequency of watching television and number of tetanus injections before pregnancy were statistically significant for maternal pregnancy body mass index in Ethiopia $(p \leq 0.05)$. Conclusion: The risk of over pregnancy weight and body mass index increased when parent prefer high number of waiting time to birth another child in Ethiopia. In addition the risk of over pregnancy weight and body mass index increased when mother received more tetanus injection during pregnancy.
\end{abstract}

Keywords: Bi-variate Linear Model, Ethiopian Demographic Health Survey, Body Mass Index, Pregnant Women

\section{Introduction}

Obesity during pregnancy may cause adverse outcomes, not only in the mother but also in the child. Low body mass index continues to be a major health burden in addition to the emergence of new competing public health priority high body mass index (BMI) in developing countries [3].

Gestational weight gain is also higher than ever before, with approximately 40 percent of pregnant women gaining more weight than its recommended [11].

In developed countries, underweight women may smoke, which may contribute to both tetanus injection before pregnancy and low birth weight, but women smoke much less often in developing countries. A low maternal BMI and sub- optimal weight gain during pregnancy are long recognized risk factors for delivery of infants too small for gestational age, LBW as well as to increase the risk of subsequent obesity and hypertension in the off- spring. In the United States of America, it was found that only 2 percent of pregnant women have a BMI less than 18.5 and more than 50 percent have a BMI greater than 25 . The most work on the effect of maternal BMI on neonatal BW has been done in developed countries. The anthropometric characteristics of women in the developed world are 
different from those of the resource poor nations. Young maternal age, low maternal BMI, and poor weight gain in pregnancy are associated with both increased risk of LBW and poor infant survival $[2,19]$.

BMI is a fairly reliable indicator of body fatness for most people. BMI does not measure body fat directly, but research has shown that BMI correlates to direct measures of body fat.

Socio-demographic factors associated with BMI classes like age, school year, practicing high competition sport, being federate in a sport or having a vegetarian diet. The educational factors associated with BMI classes included only seminars given at school by a nutritionist. Behavioral factors significantly associated with BMI included; learning in classes, playing in the open air, reading books or use of internet [10].

Study by Ronnenberg et.al, reported that maternal nutritional status is important to maternal and fetal well being, and BMI were influenced by ethnicity and genetics [17]. Nutrient intake and weight gain during pregnancy are the two main factors affecting maternal and infant outcomes [1].

Using data from a national sample of children in the U.S. and study by Datar examines family size is associated with child BMI and obesity [6]. Frequent television watching was associated with obesity among rural women of reproductive age in Myanmar [10].

Goodrich stated that women were motivated to exercise for personal health benefits but fear exercise may harm the fetus. In addition, the lack of health care provider were inconsistent with recommendations has been consistently reported as a barrier [9].

Based on national research council and others (2007) report stated that interactions among several biological factors such as pregnancy weight, age, parity, and stature influence gestational weight gain and the biological influences on gestational weight gain vary widely among women and other potential metabolic factors that may affect gestational weight gain which is placental secretions or metabolic changes in obese women remain poorly understood [6].

Magalhaes stated that the determinants of excessive weekly weight gain were family income and the prevalence rate of excessive weekly weight gain in pregnant women in the second and third trimesters was found to be 42 percent [14].

The main objective of the study was identifying the factors that affect maternal weight and body mass index among pregnant women in Ethiopia. Parts of this paper were organized as follows. Section 2 describes the materials and methods used in this study, section 3 presents the results with its discussion and conclusion and recommendations are provided in section 4 .

\section{Research Methodology}

\subsection{Descriptions of Study Population}

This research utilized Ethiopian 2016 demographic and health survey as its source of data which was the fourth comprehensive and nationally representative population and health survey. It is important feature of the data set that avails in-depth information on demographic and health aspects of households. The data were collected by the central statistical agency at the request of the ministry of health. Data collection took place from January 18, 2016 to June 27, 2016 [7]. Mothers who were pregnant and remember her maternal weight and body mass index which record from January 18, 2016 to June 27, 2016 were included in the study.

\subsection{Study Design}

Cross-sectional study was conducted to assess factors that affect maternal weight and body mass index among pregnant women in Ethiopia.

\subsection{Study Variables}

Response variable: In this study there are two response variables. Those are pregnant maternal weight and pregnant body mass index.

Independent variables: the independent variables of the study includes the variables such maternal height, preferred waiting time for birth, tetanus injection, region, desire more child, family size, maternal height, Place of residence, age, source of water supply, HIV status of mother, children ever born, frequency of watching television.

\subsection{Methods of Data Analysis}

The statistical methods used in this study were descriptive statistics (percentages and mean) and inferential statistics with bivariate multiple linear regression models).

\subsubsection{Bi-variate Multiple Linear Regression Models}

Under this study, we did bi-variate multiple linear regression models (with $\mathrm{m}=2$ response variables). The model would be multiple due to more than one predictor variables $(\mathrm{p}>1)$, with a linear function of parameters $\left(\beta_{1}, \beta_{1}, \beta_{2}, \ldots, \beta_{r}\right)$. Each response is assumed to follow its own regression model,

$$
\begin{aligned}
& Y_{1}=\beta_{01}+\beta_{11} X_{1}+\beta_{12} X_{2}+---+\beta_{r 1} X_{r}+\varepsilon_{1} \\
& Y_{2}=\beta_{02}+\beta_{12} X_{1}+\beta_{22} X_{2}+---+\beta_{r 2} X_{r}+\varepsilon_{2}
\end{aligned}
$$

The matrix notation of the model is given as:

$$
\mathrm{Y}=\mathrm{X} \beta+\varepsilon
$$

Where,

$$
Y=\left[\begin{array}{cc}
y_{11} & y_{21} \\
\vdots & \vdots \\
y_{1 n} & y_{2 n}
\end{array}\right], X=\left[\begin{array}{ccc}
x_{11} & \ldots & x_{1 r} \\
\vdots & \vdots & \vdots \\
x_{n 1} & \ldots & x_{n r}
\end{array}\right], \beta=\left[\begin{array}{cc}
\beta_{01} & \beta_{02} \\
\vdots & \vdots \\
\beta_{r 1} & \beta_{r 2}
\end{array}\right], \varepsilon=\left[\begin{array}{cc}
\varepsilon_{11} & \varepsilon_{21} \\
\vdots & \vdots \\
\varepsilon_{1 n} & \varepsilon_{2 n}
\end{array}\right]
$$

The error term has $\mathrm{E}(\varepsilon)=0$ and $\operatorname{var}(\varepsilon)=\Sigma$.

Where, 


$$
\Sigma=\left[\begin{array}{ll}
\sigma_{11} & \sigma_{12} \\
\sigma_{21} & \sigma_{22}
\end{array}\right]
$$

Thus, the error terms associated with different responses on the same trial are correlated.

In the bi-variate analysis, the dependent variable should be normally distributed within groups and also in multi-variate designs, with multiple dependent measures, the homogeneity of variances assumption described earlier also applies. However, since there are multiple dependent variables, it is also required that their inter-correlations (covariance) are homogeneous across the cells of the design.

Bi-variate multiple regression model has unknown parameters. The parameter value was obtained from parameter estimation.

According to Nkurunziza and S. Ejaz Ahmed the estimation methods mostly used are the multivariate least square estimation [16].

\subsubsection{Parameter Estimation}

The most common method for estimating bi-variate linear regression is least squares estimation (OLS). The least square for $\beta$ minimizes the sums of squares elements on the diagonal of the residual sum of squares and cross products matrix $(Y-$ $Z \hat{\beta})^{\prime}(Y-Z \hat{\beta})$. Since the matrix $(Y-Z \hat{\beta})^{\prime}(Y-Z \hat{\beta})$ has smallestpossibletrace.

Using the least squares estimator for $\beta$ we can obtain predicted values and compute residuals. From the theory of the least squares in uni-variate regression, we can get the estimator of $\beta$ by minimizing $\varepsilon^{\prime} \varepsilon$ where $Y-X \hat{\beta}$ is $n \times 2$ error matrix. We minimize $\varepsilon^{\prime} \varepsilon$ with constraints of non-negative matrix, the trace, the determinant, and the largest Eigen value, i.e. estimating $\hat{\beta}$ meet the following inequality's for all possible matrices of $\beta$ respectively.

$$
\begin{gathered}
(Y-Z \hat{\beta})^{t}(Y-Z \hat{\beta}) \leq(Y-Z \beta)^{t}(Y-Z \beta) \\
\operatorname{trace}(Y-Z \widehat{\beta})^{t}(Y-Z \widehat{\beta}) \leq \operatorname{trace}(Y-Z \beta)^{t}(Y-Z \beta) \\
\left|(Y-Z \widehat{\beta})^{t}(Y-Z \widehat{\beta})\right| \leq\left|(Y-Z \beta)^{t}(Y-Z \beta)\right| \\
\operatorname{maxeig}(Y-Z \widehat{\beta})^{t}(Y-Z \widehat{\beta}) \leq \max e i g(Y-Z \beta)^{t}(Y-Z \beta)
\end{gathered}
$$

In fact, the four criteria are equivalent to each other. Under any criteria of the four, we can get the same least square estimator of $\beta$, given by

$$
\hat{\beta}=\left(X^{\prime} X\right)^{-1} X^{\prime} Y
$$

Equation (4) is BLUE of parameters. Therefore the individual coefficients and standard errors produced by bivariate linear regression are identical to those that would be produced by regress each response against the set of independent variable separately. The difference lies in that the bi-variate linear regression as joint estimator also estimates the between equation covariance, so we can test the interrelationship between coefficients across equations.

Variety of regression models from the same set of variables could be constructed. According to David $\mathrm{J}$ and Lilja backward elimination process was prefer because it is usually straightforward to determine which factor we should drop at each step of the process [11]. Backward elimination has further advantage, in that several factors together may have better predictive power than any subset of those factors. As a result, the backward elimination process is more likely to include these factors as a group in the final model than the forward and step wise selection process. Therefore, under this study we had used backward elimination.

\section{Results and Discussion}

\subsection{Some Demographic and Socioeconomic Characteristics of the Study Subjects}

A total of 1996 pregnant were included in the study. Out of the total 1996 pregnant were included in the study, the mean of maternal BMI is $22.5 \mathrm{~kg} / \mathrm{m}^{2}$ with standard deviation $4.38 \mathrm{~kg} / \mathrm{m}$ and the average family size were 5 with standard deviation 2 approximately. Moreover, the average weights of mother were $56.52 \mathrm{~kg}$ with standard deviation $11.84 \mathrm{~kg}$. From total children ever born point of view the average total child ever born were 3 with standard deviation 2 approximately and the average height of mothers were 1.58 meter with standard deviation 0.67 meter.

In this study $44.7 \%$ mothers who watch TV at least once a week. In addition, there were $56.6 \%$ mothers who received tetanus injunction before birth between 1 and 3 times and $14.7 \%$ mothers who were not received tetanus injunction before pregnancy. Moreover, 32.3\% mothers were between 25 and 29 age group and $48.1 \%$ wants to born after more 2 years.

Furthermore, this study discussed the effect of explanatory variables when maternal body mass index and weight were fitted jointly. Under this study E-statistic or energy test of multi-variate normality that was show multivariate normality with p-value 0.25 was used. From descriptive result shows the average duration of pregnancy were 35.87 weeks with standard deviation 1.01 weeks and the average weight of new born baby were 3.27 with standard deviation 0.83 . The correlation between maternal weight and maternal body mass index is 0.92 .

\subsection{Model Assumption Diagnosis}

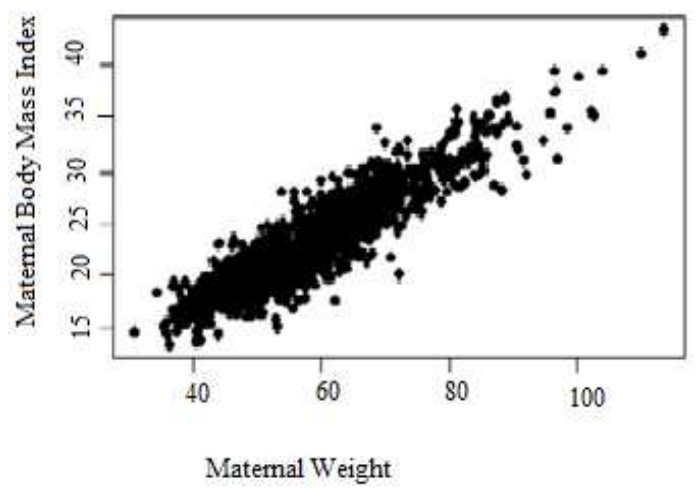

Figure 1. Plot of maternal body mass index against maternal weight. 
From scatter plot shown in Figure 1, indicate the linear relationship between maternal weight and maternal body mass index.

In addition residuals plot shown on Figure 2, seems to indicate that the residuals and the fitted values confirm linearity without distinct patterns and shows constant variance and also quintile-normal plot of Figure 3, confirms normality of errors which was residual points follow the straight dashed line. Moreover, Figure 4 shows the most observation had small cook's distance deviate from straight dashed line mean that the data set has not more influential observation.

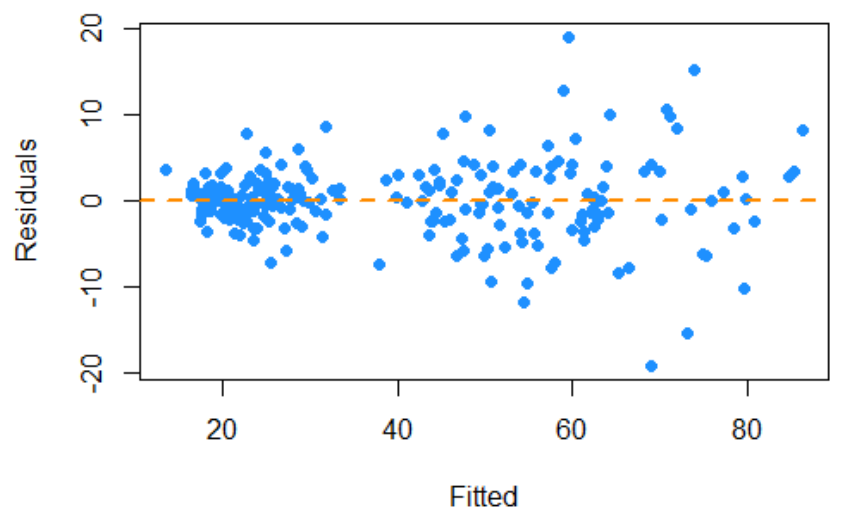

Figure2. Residual plot.

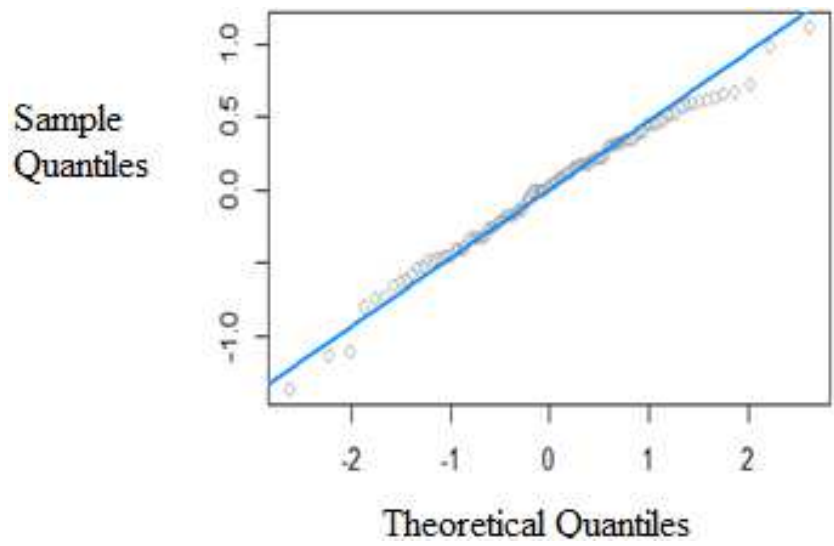

Figure3. Normal Q-Q plot.

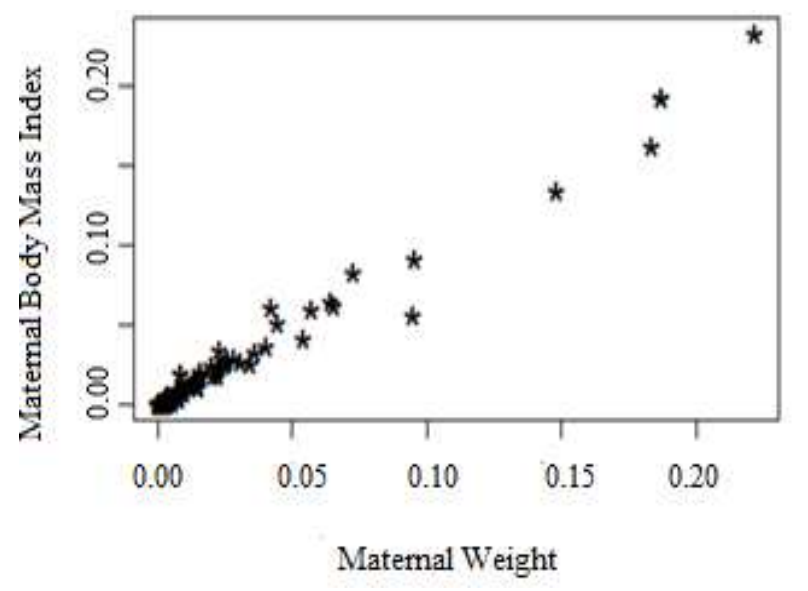

Figure 4. Plot of Cooks Distance.

\subsection{Determine of Maternal Weight and Body Mass Index: Bi-variate Multiple Linear Regression}

After the overall assumptions, bi-variate multiple linear regression model of maternal body mass index and maternal weight over the significant predictors was modeled based on estimated value of the parameter that from Table 1 . The fitted bi-variate linear regression model that relating maternal body mass index and maternal weight with the explanatory variables is given as:-

Maternal Weight $=9.43 *$ secondary $-48 *$ maternal height $14.49 *$ Preferred with 3year $-11.88 *$ Preferred with 5year $17.04 *$ Preferred with 6 year $+9.93 * 1-3$ times tetanus injection times $+13.75 * 4-6$ times tetanus injection $-10.62 *$ greater than 7 times tetanus injection+11.29*SNNP+13.97*watch Tv less than once week $+10.36759 *$ desire more child with 5 year.

And

Body Mass Index $=3.76 *$ secondary $+5.69 *$ Preferred with 3year- $4.81 *$ Preferred with 5 year.

$-6.93 *$ Preferred with 6year $+3.99 * 1-3$ times tetanus injection $+5.45 * 4-6$ times tetanus injection $-4.35 *$ greater than 7 times tetanus injection $+4.53 * \mathrm{SNNP}+5.38 *$ watch TV less than once week $+3.94 *$ desire more child with 5 year $+0.69 *$ family size.

From the model parameter of pregnancy maternal weight, the mean of pregnancy weight of mother when her husband learn up to secondary education decreased by factor 9.43 while pregnancy body massindex decreased by factor 3.76 as compared to who was no learn at all with similar effect of other co-variants. This result is in line with the previous study by Mullany [15].

In addition, the mean of pregnancy weight of mother who was waiting three, five and six year for birth of another child decreased by factor $14.49,11.88$ and 17.04 respectively as compared to waiting less than one year for birth of another child when the effect of other variable remains constant. This has been confirmed by the study [5]. Furthermore, the mean of pregnancy weight of mother who was received injections of tetanus 1 up to 3 and 4 up to 6 times before birth increased by a factor of 9.93 and 13.75 respectively as compared to who was not received injections of tetanus before birth when the effect of other variables remain constant.

But, mother who was received injections of tetanus greater than seven times before birth then, the mean of pregnancy weight of mother was decreased by factor 10.62 . This resultis also in line with the study of [4, 20].

Moreover, the mean of pregnancy weight of mother who lived in South Nation Nationality of people increased by a factor of 11.29 as compare to mother who lived in Tigray.

Furthermore, the mean of pregnancy weight of mothers who watching television for less once a week increased by factor 13.97 as compared to those not watching at all which is similar with the previous studies conducted by the scholars [17]. With the change in one centimeter in height, the mean of pregnancy weight of mother increased by 48 units.

Finally, the mean of pregnancy weight of mother who 
desire more child with 5 year increased by factor of 10.36759 as compared to those desire more child with 2 year respectively.

The model parameter of pregnancy body mass index were discussed as, the mean of pregnancy body mass index of mother who was learn up to secondary education decreased by factor 3.76 as compared to who was no learn at all when the effects of other variable remain constant. In addition, the mean of body mass index of mother who was waiting three, five and six year for birth of another child decreased by factor 5.69, 4.81 and 6.93 respectively as compared to waiting less than one year for birth of another child, which has been also confirmed by the study of Conde-Agudelo [5].

Moreover, the mean of pregnancy body mass index of mother who was received injections of tetanus 1 up to 3 and 4 up to 6 times before birth increased by a factor of 3.99 and 5.45 respectively as compared to who was not received injections of tetanus before birth. But, mother who was received injections of tetanus greater than seven times before birth, the mean of pregnancy body mass index of mother was decreased by factor 4.35 .

Furthermore, the mean of pregnancy body mass index of mother who lived in South Nation Nationality of people (SNNP) increased by a factor of 4.53 as compare to mother who lived in Tigray which is similar with the previous studies conducted by different scholars [17]. The mean of pregnancy body mass index of mother who watching television at least once a week increased by factor 5.38 as compared to those not watching at all which is in line with result of study [12].

Family size is change by one individual, then the mean of pregnancy body mass index of mother increased by 0.69 units. Similar result was observed in another study done by [8]. Finally, the mean of pregnancy body mass index of mother who desire more children with 5 year increased by factor 3.94 as compared to those desire more child with 2 year respectively.

Table 1. Results of Bi-variate Multiple Linear Regression.

\begin{tabular}{|c|c|c|c|c|c|}
\hline \multicolumn{3}{|c|}{ Pregnancy Maternal Weight } & \multicolumn{3}{|c|}{ Pregnancy body mass index } \\
\hline Parameter & Estimate & P value & Parameter & Estimate & P value \\
\hline \multicolumn{3}{|c|}{ Husband Education (no=ref) } & \multicolumn{3}{|c|}{ Husband Education (no=ref) } \\
\hline Secondary & -9.34 & $0.029 *$ & Secondary & -3.76 & $0.030 *$ \\
\hline \multicolumn{3}{|c|}{ Tetanus injection $(\mathrm{no}=\mathrm{ref})$} & \multicolumn{3}{|c|}{ Tetanus injection (no=ref) } \\
\hline $1-3$ times & 9.93 & $0.022 *$ & 1-3times & 3.99 & $0.021 *$ \\
\hline 4-6 times & 13.75 & $0.005 * *$ & 4-6 times & 5.45 & $0.005 *$ \\
\hline$>7$ times & 10.62 & $0.043^{*}$ & $>7$ times & 4.35 & $0.038^{*}$ \\
\hline \multicolumn{3}{|l|}{$\begin{array}{l}\text { Waiting time for } \\
\text { birth }(<1 \text { year=ref) }\end{array}$} & \multicolumn{3}{|l|}{$\begin{array}{l}\text { waiting time for } \\
\text { birth }(<1 \text { year=ref })\end{array}$} \\
\hline Preferred (3year) & -14.49 & $0.009 * *$ & Preferred ( 3 year) & -5.69 & $0.009 * *$ \\
\hline Preferred (5year) & -11.88 & $0.020^{*}$ & Preferred (5 year) & -4.81 & $0.019^{*}$ \\
\hline Preferred (6year) & -17.04 & $0.002 * *$ & Preferred (6 year) & -6.93 & $0.002 * *$ \\
\hline \multicolumn{3}{|l|}{ Region (Tigray=ref) } & \multicolumn{3}{|l|}{ Region (Tigray $=\mathrm{ref}$ ) } \\
\hline \multicolumn{3}{|l|}{ SNNP 11.29 0.034* } & \multicolumn{3}{|l|}{ SNNP 4.53 0.034* } \\
\hline \multicolumn{3}{|l|}{ Watch Tv (no=ref) } & \multicolumn{3}{|l|}{ Watch $\operatorname{Tv}($ no=ref) } \\
\hline Less than once a week & 13.97 & $0.005 * *$ & Less once a week & 5.38 & $0.007 * *$ \\
\hline Maternal height & 48 & $0.048 *$ & Family size & 0.69 & $0.033^{*}$ \\
\hline \multicolumn{3}{|c|}{ Desire child (with 2 year=ref) } & \multicolumn{3}{|c|}{ Desire child (with 2 year=ref) } \\
\hline Desire (with5 year) & 10.368 & $0.034 *$ & Desire (with 5 year) & 3.94 & $0.043 *$ \\
\hline \multicolumn{3}{|l|}{$\begin{array}{l}R^{2}=0.82 \\
\text { Adiusted } R^{2}=0.80\end{array}$} & \multicolumn{3}{|l|}{$R^{2}=0.79$} \\
\hline
\end{tabular}

*significant at $5 \%$ level of significance, ${ }^{* *}$ significant at $1 \%$ and at $5 \%$ level of significance (ref) is reference category

\section{Conclusion and Recommendations}

\subsection{Conclusion}

This study was held with the objective of determine the determinant of pregnancy weight and body mass index among pregnant women in Ethiopia simultaneously to make recommendation for concerned body. Therefore, this study concluded that preferred waiting time for birth, number of tetanus injections before pregnancy, husband education level, region, watch television, desire more child are statistically significant at $5 \%$ level of significance for pregnancy weight and body mass index in Ethiopia jointly.

The risk of overweight pregnancy and high body mass index increased when parent prefer high number of waiting time to birth another child in Ethiopia. In addition the risk of over pregnancy weight and body mass index increased when mother received more tetanus injection during pregnancy.

Furthermore, the risk of over pregnancy weight and body mass index increased when parent was not educated, desire more child wants with more than 2 years expectancy.

\subsection{Recommendations}

Based on the finding of the study the following recommendations were forwarded

i. The health center of the country should work on the perception of women in order to enhance pregnancy weight and body mass index.

ii. Education level of husband plays an important role in minimizing pregnancy weight and body mass index. Thus, the respective bodies' needs to focus on sustaining education implemented to reduce weight and body mass index. 
iii. Since the risk of over pregnancy weight and body mass index increased when mother received more tetanus injection during pregnancy, health professionals should care on pregnant woman during tetanus injection.

iv. This research result helps to policy-makers and programmer to design ways of control the over pregnancy weight and body mass index in Ethiopia.

\section{Abbreviations}

BLUE: Best Linear Unbiased Estimator, BMI: Body Mass Index, kg: kilogram, Maxeig: Maximum Eigen values, LBW: Low Birth Weight, m: Meter , $R^{2}$ : Coefficient of determination, SNNP: South Nation and National People, TV/tv: Television

\section{Availability of Data and Materials}

The dataset used and analyzed during this study are available from the corresponding author on reasonable request.

\section{Conflict of Interest}

The authors declare that they have no conflict of interests.

\section{Authors' Contributions}

MA was involved in the conception and design of the study, data collection and entry, interpretation and drafting the manuscript. KK was involved in the design and review of protocol development, interpretation of the data and review of the manuscript. All authors read and approved the final manuscript.

\section{Appendix}

\author{
$R$ Codes for the Analysis \\ attach (data) \\ names (data) \\ $M=$ as.matrix (data[,c(9,19)]) \\ summary(maternal_weight,NeBMI) \\ $m a t=c(\operatorname{var}($ maternal_weight $), \operatorname{var}(N e B M I))$ \\ $s d=\operatorname{sqrt}($ mat $)$ \\ prop.table(table(wealth_index)) \\ round(100*prop.table(table(wealth_index)),1) \\ library (MASS) \\ $\operatorname{library}(M V N)$ \\ mvnal<-mvn $($ data $=M, \quad$ mvnTest=ënergy,"univariatePlot \\ $=\ddot{q} q$ plot $)^{\prime \prime}$ \\ mvna $2<-m v n($ data $=M, \quad$ mvnTest=ënergy,"univariatePlot \\ $=$ histogram" \\ corlat<-cor(maternal_weight,NeBMI) \\ plot(maternal_weight,NeBMI, xlab=thaternal_weight ," \\ ylab= thaternal body mass index,"pch=19) \\ Model $=\operatorname{lm}(m \sim$ timing + as.factor(wealth_index $)+$ as. factor $(n$ \\ umber_befbirth) +as.factor(number_befpre) + as.factor(Regio \\ n) + as.factor(new_sourwat) + as.factor(freq_watch_television)
}

+total_child_everborn+as.factor(alchool)+familiy_size+as.f actor(husband_partner_edu_le)+maternal_height+as.factor( Preferred) + as.factor(age group) + as. factor(desire)) plot(fitted(Model), resid(Model), col = dodgerblue,"pch = 20, cex $=1.5, x l a b=$ Fitted,"ylab $=$ Residuals" abline $(h=0$, lty $=2$, col = "darkorange,"lwd = 2) qqnorm(resid(Model),col = 'darkgrey)' qqline(resid(Model), col = alodgerblue,"lwd = 2) cooksd <-cooks.distance(Model) plot(cooksd, pch=",cex=2)

\section{References}

[1] Nilufer Akgun, Huseyin L Keskin, Isık Ustuner, Gulden Pekcan, and Ayse F Avsar. 2017. Factors affecting pregnancy weight gain and relationships with maternal/fetal outcome in Turkey. Saudi medical journal. Vol. 38, No. 5, 2017, pp. 503508. doi: 10.15537/smj.2017.5.19378.

[2] Radha Y Aras et al. 2013. Is maternal age risk factor for low birth weight? Archives of medicine and health sciences. Vol. 1, No. 1, 2013, pp. 33. doi: 10.4103/2321-4848.113558.

[3] Yolan Banda, Victoria Chapman, Robert L Goldenberg, Benjamin H Chi, Sten H Vermund, and Jeffrey SA Stringer. 2007. Influence of body mass index on pregnancy outcomes among HIV-infected and HIV-uninfected Zambian women. Tropical Medicine \& International Health. Vol. 12, No. 7, 2007, pp. 856-861. doi: 10.1111/j.1365-3156.2007.01857.x.

[4] Helen Y Chu, Janet A Englund. 2014. Maternal immunization. Clinical Infectious Diseases. Vol. 59, No. 4, 2014, pp. 560 568. doi.org/10.1093/cid/ciu327

[5] Agustin Conde-Agudelo, Anyeli Rosas-Bermu'dez, Ana C Kafury-Goeta. 2007. Effects of birth spacing on maternal health: a systematic review. American journal of obstetrics and gynecology. Vol. 196, No. 4, 2007, pp. 297-308. doi: 10.1016/j.ajog.2006.05.055.

[6] National Research Council et al. 2007. Influence of pregnancy weight on maternal and child health: workshop report. National Academies Press.

[7] Central Statistical Agency (CSA) and ICF International. 2012. "Ethiopia demographic and health survey 2011". Addis Ababa, Ethiopia and Calverton, Maryland, USA: Central Statistical Agency and ICF International.

[8] Ashlesha Datar. 2017. The more the heavier? Family size and childhood obesity in the U.S. Social Science \& Medicine. Vol. 180, 2017, pp. 143-151. doi.org/10.1016/j.socscimed.2017.03.035

[9] Kara Goodrich, Mary Cregger, Sara Wilcox, Jihong Liu. 2013. A qualitative study of factors affecting pregnancy weight gain in African American women. Maternal and child health journal. Vol. 17, No. 3, 2013, pp. 432-440. doi: 10.1007/s10995-012-1011 1.

[10] Raquel PF Guin'e, Sofia R Fernandes, Jos'e Lu'is Abrantes, Ana Paula Cardoso, Manuela Ferreira. 2016. Factors affecting the body mass index in adolescents in Portuguese schools. Croatian Journal of Food Technology, Biotechnology and Nutrition Vol. 11, No. 2, 2016, pp. 58-64 
[11] Erica P Gunderson. 2009. Childbearing and obesity in women: weight before, during, and after pregnancy. Obstetrics and Gynecology Clinics. Vol. 36, No. 2, 2009, pp. 317-332.

[12] Rajat Das Gupta, Ibrahim Hossain Sajal, Mehedi Hasan, Ipsita Sutradhar, Mohammad Rifat Haider, Malabika Sarker. 2019. Frequency of television viewing and association with overweight and obesity among women of the reproductive age group in Myanmar: results from a nationwide cross-sectional survey. BMJ open Journal. Vol. 9, No. 3, 2019. doi.org/10.1136/bmjopen-2018-024680.

[13] David J Lilja. 2016. Linear Regression Using R: An Introduction to Data Modeling. University of Minnesota, Libraries Publishing.

[14] Elma Izze da Silva Magalh aes, Daniela Santana Maia, Carla Fabr'icia Arau'jo Bonfim, Michele Pereira Netto, Joel Alves Lamounier, Daniela da Silva Rocha. 2015. Prevalence and factors associated with excessive weight gain in pregnancy in health units in the southwest of Bahia. Brazilian Journal of Epidemiology. Vol. 18, No. 4, 2015, pp. 858-869. doi: 10.1590/1980-5497201500040014

[15] Britta C Mullany, Stanley Becker, MJ Hindin. 2006. The impact of including husbands in antenatal health education services on maternal health practices in urban Nepal: results from a randomized controlled trial. Health education research. Vol. 22, No. 2, 2006, pp. 166-176. doi: 10.1093/her/cyl060.

[16] S'ev'erien Nkurunziza, S Ejaz Ahmed. 2011. Estimation strategies for the regression coefficient parameter matrix in multivariate multiple regression. Statistica Neerlandica. Vol. 6, No. 4, 2011, pp. 387-406. doi.org/10.1111/j.14679574.2011.00491.x.

[17] Alayne G Ronnenberg, Xiaobin Wang, Houxun Xing, Chanzhong Chen, Dafang Chen, Wenwei Guang, Aiqun Guang, Lihua Wang, Louise Ryan, Xiping Xu. 2003.Low preconception body mass index is associated with birth outcome in a prospective cohort of Chinese women. The Journal of nutrition. Vol. 133, No. 11, 2003, pp. 3449-3455. doi: 10.1093/jn/133.11.3449.

[18] Abdul Sattar, Shahbaz Baig, Naveed UR Rehman, Muhammad Badar Bashir. 2013. Factors affecting BMI. The Professional Medical Journal. Vol. 20, No. 6, 2013, pp. 956964.

[19] Swati Singh, Constance E Shehu, Daniel C Nnadi, et al. 2016. The relationship between maternal body mass index and the birth weight of neonates in north-west Nigeria. Sahel Medical Journa. Vol. 19, No. 4, 2016, pp. 185-189. doi: 10.4103/11188561.196359 .

[20] Tadese Ejigu Tafere, Mesganaw Fanthahun Afework, Alemayehu Woreku Yalew. 2018. Providers adherence to essential contents of antenatal care services increases birth weight in Bahir Dar city administration, North West Ethiopia: a prospective follow up study. Reproductive health. Vol. 15, 2018, No. 163. doi.org/10.1186/s12978-018-0610-8. 